\title{
Influences of weather phenomena on automotive laser radar systems
}

\author{
R. H. Rasshofer ${ }^{1}$, M. Spies ${ }^{2}$, and H. Spies ${ }^{2}$ \\ ${ }^{1}$ BMW Group Research and Technology, Hanauer Str. 46, 80992 Munich, Germany \\ ${ }^{2}$ Ingenieurbüro Spies, Seibersdorf 18, 86558 Hohenwart, Germany
}

\begin{abstract}
Laser radar (lidar) sensors provide outstanding angular resolution along with highly accurate range measurements and thus they were proposed as a part of a high performance perception system for advanced driver assistant functions. Based on optical signal transmission and reception, laser radar systems are influenced by weather phenomena. This work provides an overview on the different physical principles responsible for laser radar signal disturbance and theoretical investigations for estimation of their influence. Finally, the transmission models are applied for signal generation in a newly developed laser radar target simulator providing - to our knowledge - worldwide first HIL test capability for automotive laser radar systems.
\end{abstract}

\section{Introduction}

Advanced driver assistance systems (ADAS) develop from comfort enhancement to safety applications. With a constantly growing need for higher perception sensor data quality, laser-based sensors tend to dominate many experimental intelligent vehicle systems with applications ranging from pedestrian protection (see Walchshäusl et al., 2006) to fully autonomous driving (see Darms et al., 2009). New, promising signal processing approaches like e.g. occupancy grid based methods adopted from robotics (see Thrun et al., 2005) heavily rely on laser radar sensors and pave the way for highly automated driver assistance. Compared to millimeterwave radar, laser radar (lidar) systems provide higher angular resolution in the azimuth plane being able to separate targets spaced less than 1 deg apart. This is a key feature for many ADAS applications since high angular resolution is crucial for the determination of object width and shape. This information provides valuable input to object classification algorithms and tracking systems that precisely determine

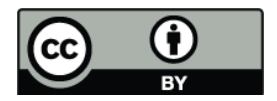

Correspondence to: $\mathrm{M}$. Spies (martin.spies@ib-spies.de) the object's orientation. Moreover, laser radar systems using more than one detection layer are providing angular resolution even in elevation. This feature is especially important for automatic alignment procedures, for robust pitch angle compensation, for lane marking detection and for estimation of object height. Although measurement capability in elevation is theoretically possible with millimeter-wave radar, too, no such products have shown up on the automotive radar market so far. This is mostly due to the very high complexity of radar front-ends exhibiting two-dimensional millimeterwave beam steering. Although from a performance point of view, laser radar seems to be ideally suited for use in ADAS, its sensitivity to weather phenomena such as fog, rain or snow has been considered a major drawback for these perception sensors and limited their use on the mass market to low-cost systems. This work deals with the primary physical effects influencing laser radar systems under bad weather conditions. From laser radar theory, we derive a model for the raw signals to be expected and subsequently estimate the performance degradation. Moreover, the developed signal model is used for signature generation and verification in a novel laser radar HIL test system which provides for the first time the possibility to evaluate and optimize weather-based influences on laser radar performance in the laboratory in real time.

\section{Laser radar fundamentals}

\subsection{Target detection}

Laser radar systems, sometimes called LADAR (Laser radiation detection and ranging), LIDAR (light detection and ranging) and LIVAR (laser-illuminated viewing and ranging) use a modulated, intense source of light to illuminate the sensor's field of view containing objects to be detected. From the objects in the sensor's field of view, a certain part of optical power is reflected back towards the sensor where the signal is detected by an optical receiver. In most automotive laser radar systems, optical signal transmitters and receivers are

Published by Copernicus Publications on behalf of the URSI Landesausschuss in der Bundesrepublik Deutschland e.V. 
located close to each other or even use the same optics in a coaxial beam configuration. For successful detection, the received optical signal has to be larger than the sensitivity limit of the optical receiver. For automotive applications, only incoherent detection has been realized so far. As optical receivers, PIN-photo diodes (PIN doping profile: $p^{+}, i$ (intrinsic), $n$ ) and avalanche photo diodes (short: APD) typically are used. Both types of diodes feature higher bandwidth than standard $p n$-photo diodes. Compared to PIN-diodes, APDs usually provide a superior signal-to-noise ratio (SNR) due to their intrinsic gain, however require complicated biasing (see Smith et al., 1978).

\subsection{Transmitter design}

Eye safe operation is a must for automotive laser radar systems. As these systems should be laser class 1 or class $1 \mathrm{M}$ products, their design has to comply with the rules given in IEC 60825-1:2007 (2007) which limit the peak power of a single pulse and the average power depending on field of view, pulse burst rate and lens aperture. Since most automotive laser radar systems use semiconductor laser diodes as optical transmitters, the system's wavelength is either $0.850 \mu \mathrm{m}$ or $0.905 \mu \mathrm{m}$. For these wavelengths, low-cost pulse laser diodes and low-noise APDs are readily available.

\subsection{Range measurement}

Due to the modulation of the transmitted signal, the time difference $t$ (sometimes called TOF time of flight) between illumination signal emission and detection of the signal reflected by the target can be measured. Since $t$ is directly proportional to the distance the signal traveled, the range $R$ of the reflection point can be deduced, assuming $c$ to be the known propagation velocity of the optical signal in the given transmission medium.

$R=c t / 2$.

From fundamental pulse radar physics (see e.g. Skolnik, 1990 ), it is known that the system's range resolution $\delta R$, i.e. the capability of the laser radar to separate two detections which are located at a radial distance $\delta R$ from each other within one beam - is limited by the bandwidth of the transmitted signal and the bandwidth of the optical detector.

\subsection{Angular measurement}

To measure the angle between sensor and reflection point, different techniques can be applied. Most laser radar sensors are using a sharply limited illumination beam which only illuminates an area limited by $\pm \vartheta_{\mathrm{T}}$ in elevation and $\pm \varphi_{\mathrm{T}}$ in azimuth. The receiver's optics, however uses a projection only observing a sharply limited detection area characterized by $\pm \vartheta_{R}$ in elevation and $\pm \varphi_{R}$ in azimuth. Object detection can only take place if a reflection point is located within the illumination beam of the transmitter and the detection area of the receiver. The projection of both the illumination beam and the reception area typically is not an ellipse, however. A rectangular assumption can be used in most cases in order to enable analytic calculation as shown by Spies and Spies (2006b). In most laser radar sensors, the field-of-view is divided into $N$ detection sectors. In multi-beam systems, the sectors are illuminated by a number of $N_{\mathrm{T}}$ transmitters and observed by $N_{\mathrm{R}}$ receivers. For coaxial optics using the same optical path both for transmit and receive, $\vartheta_{\mathrm{T}}=\vartheta_{\mathrm{R}}$ and $\varphi_{\mathrm{T}}=\varphi_{\mathrm{R}}$ yields optimal performance. For bistatic configurations, offsets and differences between illuminated area and detection area have to be taken into account in the vicinity of the sensor as will be shown in Sect. 3.3.

To cover the complete field of view, two basic system types have been developed: systems using mechanical scanning of the detection area (so called "laser scanners") and systems using multiple transmitters and receivers (so called "multibeam laser radar" or "staring laser radar"). Laser scanners tend to provide higher angular resolution than multi-beam laser radar, however due to their larger mechanical complexity often exhibit higher system costs and larger package size.

For identification of ground clutter, to compensate for horizontal misalignment and for target classification, some laser scanners use multiple layer techniques to provide some degree of resolution in elevation as stated by Spies and Spies (2006a).

\subsection{Velocity measurement}

With incoherent light detection typically used in automotive laser radar systems, a direct measurement of a target's velocity due to the DOPPLER effect is not possible. Coherent detection, often used in military DOPPLER laser radar (see Owens, 1969) is not feasible for automotive use since the coherence length of the semiconductor lasers employed by all sensors on the market today is not large enough to enable low-noise carrier frequency DOPPLER measurement. Today, velocity determination typically is performed by tracking of reflection points' movements over time. For optimized tracking, data update rates well above $15 \mathrm{~Hz}$ are necessary to cope with the dynamic requirements of future driver assistant systems. Attention has to be paid to the selection of the reflection points to be tracked. Sudden jumps of reflection points of periodic structures within the sensor's field of view tend to introduce velocity measurement errors. Moreover, for the detection of object velocity, also the shape of the object has to be taken into account. Especially in the vicinity of the sensor, laser radar often tends to see more than one reflection point on the surface of extended objects like e.g. cars. In this case, shape-based tracking techniques (see Kirchner and Ameling, 2000) like e.g. L-Shape tracking (see Fayad and Cherfaoui, 2007) have to be applied to obtain valid and precise results for lateral and longitudinal velocity of the tracked objects. 
(a)

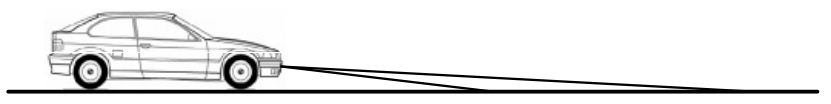

(b)

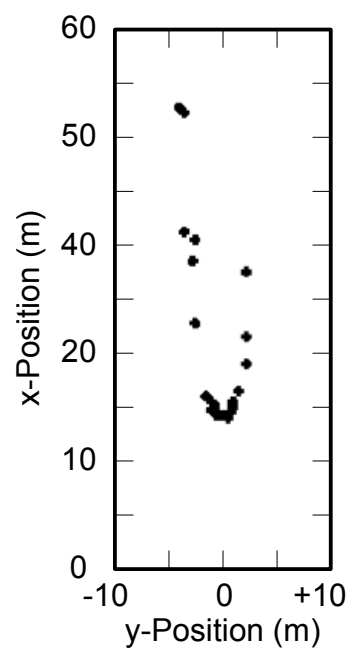

(c)

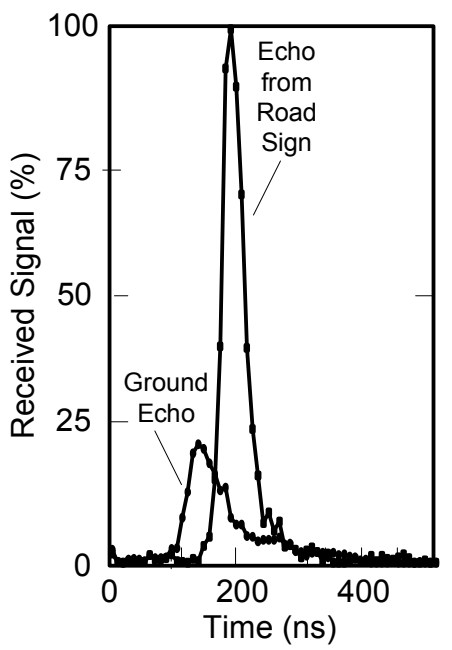

Fig. 1. (a) Beam configuration leading to detection of ground echo. (b) Typical spatial distribution ground echo detection, basis of shape-based ground echo classification. (c) Time domain classification of ground echo based on different time domain response of rigid targets (e.g. road signs, other cars etc.) and ground signal with different time domain response as shown by Issa (2007).

\subsection{Raw data based target classification}

Besides target detection and location, classification based on raw reflection data has become very important in many driver assistance and active safety systems. Generally speaking, the following classes of raw detections have to be distinguished:

Type 1. Raw detections from solid objects like cars, pedestrians or other infrastructure. In this case, the scattering plane has almost no depth-/range distribution.

Type 2. Raw detections from noise in the optical detector or detector amplifier chain. These detections can be dealt with by the application of constant false alarm rate (CFAR) techniques in the target detector, residual detections will be of statistical nature and thus will be eliminated in the tracker.

Type 3. Raw detections from electromagnetic interference (EMI) from inside or outside of the sensor e.g. power supply spikes, clock interference, laser diode modulator crosstalk etc. These detections must be carefully resolved on the hardware level by proper system design and sophisticated mechanical setups including highly effective shielding techniques. Especially periodic interference tends to be hard to deal with on a system level since these kinds of detections will not be removed in the tracker because of their periodic, deterministic nature.

Type 4. Raw detections from large signal effects in the receiver chain. These detections arise from clipping or ringing in the re-

ceive amplifier chain after application of very large optical receive signals which might be due to e.g. retro-reflecting objects. To minimize these detections, gain control in the receive amplifier chain and/or control of the transmit power must be utilized.

Type 5. Targets due to ground echo/ground clutter, often generated by the car's pitch or by bumpy roads. Since these detections do come from real world reflections, their classification must be based on additional information. If a multi layer laser radar sensor is used, a comparison between detections in different layers might be used to identify the ground echo. If the laser radar exhibits only a single layer, two methods of classification are known as described in the the work of Issa (2007): a shape-based approach, taking advantage of the special location of the ground echo detections which is based on the beam geometry and the density distribution of the transmitters (e.g. detections matching parabolic shape, see Fig. 1b and ground echo recognition based on the distributed nature of the reflection points like shown in Fig. 1.

Type 6. Detections generated by reflections in rain, fog, dust or snow. The following sections will show how the return signals for these kinds of detections look like.

Automotive laser radar sensors have to distinguish between the different types of the raw detections listed above and should only pass detections of type 1 to the subsequent clustering and tracking algorithms. All other detections should either be minimized by system design or should be clearly recognized as not relevant for the driver assistance function, providing valuable information for the subsequent signal processing (e.g. data association and tracking) Many laser radar systems show deficiencies within this classification so careful evaluation has to take place during the sensor test. Especially detections of type 6 are hard to test since environmental test conditions like rain, snow or fog are difficult to reproduce under lab conditions.

\section{Modeling of laser radar performance}

\subsection{Review of the laser radar equation}

The laser radar equation describes the received power $P_{\mathrm{R}}(R)$ at a laser radar's detector as a function of a target's range. For non-elastic scattering, the received signal power can be calculated as a convolution between the transmit signal $P_{\mathrm{T}}$ and the spatial impulse response $H(R)$ of the optical channel and the target as stated e.g. by Zhao et al. (1988):

$$
P_{\mathrm{R}}(R)=C_{A} \int_{t^{\prime}=0}^{2 R / c} P_{\mathrm{T}}\left(t^{\prime}\right) H\left(R-c t^{\prime} / 2\right) d t^{\prime}
$$

The system constant $C_{A}$ is not depending on range or time and is given by:

$C_{A}=c \eta A_{\mathrm{R}} / 2$.

$A_{\mathrm{R}}$ represents the aperture area of the sensor's optical receiver and $\eta$ characterizes losses in the receiver's optic. 
In the subsequent sections, a detailed review of all factors appearing in Eq. (2) will be carried out, considering automotive application's constraints.

\subsection{Transmit pulse modeling}

In classic laser radar theory, a DIRAC-shaped transmit pulse with a total pulse energy $E_{\mathrm{P}}$ according to Eq. (4) is assumed:

$P_{\mathrm{T} \delta}(t)=E_{\mathrm{P}} \delta(t)$

Using the rather simple transmission pulse model given in Eq. (4), the classic laser radar equation is obtained:

$$
\begin{aligned}
P_{\mathrm{R} \delta}(R)= & C_{A} \int_{t^{\prime}=0}^{2 R / c} E_{\mathrm{P}} \delta\left(t^{\prime}\right) H\left(R-c t^{\prime} / 2\right) d t^{\prime} \\
& =C_{A} E_{\mathrm{P}} H(R) .
\end{aligned}
$$

This model is valid for target ranges $R \gg c \tau_{\mathrm{P}}$ with $\tau_{\mathrm{P}}$ denoting the duration of the transmit pulse.

In most automotive sensor systems found on the market today, $\tau_{\mathrm{P}}$ is in the range of 10 to $20 \mathrm{~ns}$ resulting in a geometrical pulse length $c \tau_{\mathrm{P}}$ of 3 to $6 \mathrm{~m}$. As the sensitivity of automotive laser radar systems to fog and rain is crucial, a sound understanding of particle scattering in the range from 5 to $15 \mathrm{~m}$ in front of the laser radar is of special importance. For this target range, the simple laser radar equation is not valid and a careful evaluation of Eq. (2) has to be carried out.

A simple but yet realistic model for the time signature $P_{\mathrm{T}}(t)$ of automotive lidars' transmit pulses is a $\sin ^{2}$-function according to Eq. (6):

$P_{\mathrm{T}}(t)= \begin{cases}P_{0} \sin ^{2}\left(\frac{\pi}{2 \tau_{H}} t\right) & 0 \leq t \leq 2 \tau_{H} \\ 0 & \text { else }\end{cases}$

This model is valid for most semiconductor laser diodes powered by state-of-the-art driver circuits. $P_{0}$ denotes the peak power of the laser radar pulse and $\tau_{\mathrm{H}}$ its half-power pulse width. The total energy $E_{\mathrm{P}}$ of a single $\sin ^{2}$-impulse as defined by Eq. (6) is:

$E_{\mathrm{P}}=P_{0} \tau_{\mathrm{H}}$

Typical values of today's pulse laser radar systems exhibit a peak power $P_{0}$ of up to $80 \mathrm{~W}$ resulting in pulse energy up to $1.6 \mu \mathrm{J}$.

A generic model for the pulse shape of laser radar transmit signals is given in Steinvall et al. (2006) and analyzed for its impact on laser radar's performance.

\subsection{Spatial impulse response function}

The spatial impulse response function $H$ can be split into two functions describing the spatial impulse responses of the

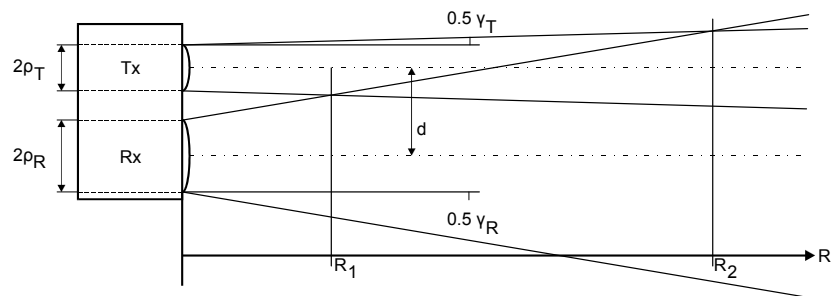

Fig. 2. Laser radar using a bistatic beam configuration.

optical channel $H_{\mathrm{C}}$ and the spatial impulse response of the target $H_{\mathrm{T}}: H=H_{\mathrm{C}} H_{\mathrm{T}}$.

The channel's spatial impulse response $H_{\mathrm{C}}$ is modeled as

$H_{\mathrm{C}}(R)=\frac{T^{2}(R)}{R^{2}} \xi(R)$.

In Eq. (8) $T(R)$ represents the total one-way transmission loss introduced by losses in the transmission medium:

$T(R)=\exp \left(-\int_{r=0}^{R} \alpha(r) d r\right)$

$\alpha(R)$ represents the local extinction coefficient characterizing the local, differential power loss in a distance $R$. Details on the calculation of $\alpha(R)$ in fog, rain or snow will be given in Sects. 3.5 and 3.6.

In Eq. (8), $\xi(R)$ describes the crossover function defined by Halldorsson and Langerhoic (1978) or Sassen and Dodd (1982) as ratio between the area $A_{\mathrm{T}}$ illuminated by the transmitter and the area $A_{\mathrm{R}}$ observed by the optical receiver:

$\xi(R)= \begin{cases}\frac{A_{\mathrm{R}}(R) \cap A_{\mathrm{T}}(R)}{A_{\mathrm{T}}(R)} & \text { if } A_{\mathrm{R}}(R) \cap A_{\mathrm{T}}(R)<A_{\mathrm{T}}(R) \\ 1 & \text { else. }\end{cases}$

In case of a coaxial transmit/receive optics, $\xi(R)$ is a constant factor not depending on range.

In the important case of bistatic beam configuration using circular illumination and detection areas with parallel optical axis exhibiting a displacement $d$, the radius $R_{1}$ of first contact between transmit and reception area is given by

$R_{1}=\frac{d-\rho_{\mathrm{T}}-\rho_{\mathrm{R}}}{\tan \left(\gamma_{\mathrm{T}} / 2\right)+\tan \left(\gamma_{\mathrm{R}} / 2\right)}$

Here, $\rho_{\mathrm{T}}$ and $\rho_{\mathrm{R}}$ denote the radius of the transmission and reception aperture and $\gamma_{T}$ and $\gamma_{T}$ the total opening angle of the transmitter's and detector's field of view, respectively. For a distance larger than $R_{2}$, the transmit- and reception area overlap completely which yields $\xi(R)=1$ for $R>R_{2}$.

$R_{2}=\frac{d-\rho_{\mathrm{R}}+\rho_{\mathrm{T}}}{\tan \left(\gamma_{\mathrm{R}} / 2\right)-\tan \left(\gamma_{\mathrm{T}} / 2\right)}$

It is clear that in this kind of beam configuration, $\xi(R)$ varies strongly with the distance. Defining

$r_{\mathrm{T}}=R \tan \left(\gamma_{\mathrm{T}} / 2\right)+\rho_{\mathrm{T}}$ and $r_{\mathrm{R}}=R \tan \left(\gamma_{\mathrm{R}} / 2\right)+\rho_{\mathrm{R}}$ 
$\phi_{\mathrm{T}}=2 \arccos \left(\frac{r_{\mathrm{T}}^{2}-r_{\mathrm{R}}^{2}+d^{2}}{2 d r_{\mathrm{T}}}\right)$

$\phi_{\mathrm{R}}=2 \arccos \left(\frac{r_{\mathrm{R}}^{2}-r_{\mathrm{T}}^{2}+d^{2}}{2 d r_{\mathrm{R}}}\right)$

the crossover function $\xi(R)$ is given as

$\xi(R)= \begin{cases}0 & \text { if } R \leq R_{1} \\ \frac{r_{\mathrm{T}}^{2}\left(\phi_{\mathrm{T}}-\sin \phi_{\mathrm{T}}\right)+r_{\mathrm{R}}^{2}\left(\phi_{\mathrm{R}}-\sin \phi_{\mathrm{R}}\right)}{2 \pi r_{\mathrm{T}}^{2}} & \text { if } R_{1}<R<R_{2} \\ 1 & \text { if } R \geq R_{2}\end{cases}$

Please note that this simplified calculation is based on a homogeneous intensity distribution over transmit beam. If laser radar systems with more complex beam configurations have to be analyzed, the same calculation steps might be used, however $\xi(R)$ might not always be available in closed form.

\subsection{Modeling hard targets}

Properties of laser radar targets are described by the spatial impulse response function $H_{\mathrm{T}}(R)$. Laser radar pulses reflected at plain surfaces of solid objects are said to hit a hard target if the time signature of the laser pulse is not changed upon reflection. In this case, a simple model for the hard target spatial impulse response $H_{\mathrm{T} \delta}(R)$ can be derived. A hard target located at $R=R_{0}$ featuring a target area of $A_{\mathrm{TA}}$ exhibits a spatial impulse response $H_{\mathrm{T} \delta}$ given as:

$H_{\mathrm{T} \delta}(R)= \begin{cases}\beta_{0} \delta\left(R-R_{0}\right) & \text { if } A_{\mathrm{TA}} \geq A_{\mathrm{T}}\left(R_{0}\right) \\ \beta_{0} \delta\left(R-R_{0}\right) \frac{A_{\mathrm{TA}}}{A_{\mathrm{T}}} & \text { if } A_{\mathrm{TA}}<A_{\mathrm{T}}\left(R_{0}\right) .\end{cases}$

$A_{\mathrm{T}}\left(R_{0}\right)$ denotes the cross-sectional area of the transmit beam at $R_{0}$ and $\beta_{0}$ describes the differential reflectivity of the target. In the important case of LAMBERT reflection characteristic with a reflectivity of $0<\Gamma \leq 1$, it is given by:

$\beta_{0}=\Gamma / \pi$.

In laser radar systems featuring very high range resolution, solid objects might not appear as hard laser radar targets if the laser beam - due to its lateral expansion - is hitting points of different range on the solid object. Errors introduced to range measurements by this effects have been analyzed by Grönwall et al. (2007) but might be considered of lower importance for automotive laser radar systems on the market today.

Depending on the geometry of the target, the angle of incident and the target's surface properties, the energy reflected by the target's surface has different angular distributions. Concerning the automotive environment, three main characteristics of angular power distribution can be found:
Type 1. Speckling reflections on flat, clean surfaces like glass or polished acrylic lacquer. Here, most of the energy is not reflected back to the laser radar receiver. This effect tends to largely increase laser radar target reflectivity variation and can be held responsible for target loss even for small target distances along with false detections due to unintended reflections from the environment.

Type 2. Diffuse reflections from e.g. dirty sheet metal or the road surface. These reflections can be modeled by a LAMBERT reflection characteristic.

Type 3. Retro reflection taking place at tail lights, license plates or road signs. In this case, almost all incident energy is directly reflected back to the location of the laser radar receiver. This kind of reflection usually yields best laser radar long-range performance but might overload or saturate the laser radar receiver chain for short range targets. In case of retro reflection, the target's reflectivity $\Gamma$ might become significantly larger than 10 .

Real world traffic objects usually represent a mixture of these 3 types of reflection characteristics. A generalized model for the angular energy distribution of reflected laser radar pulses is given by the bidirectional reflection distribution function (BRDF).

\subsection{Influences of rain and fog}

Soft laser radar targets provide distributed scattering of the transmit pulse. Their spatial impulse response function $H_{\mathrm{T}}$ is described by the backscattering coefficient $\beta(R): H_{\mathrm{T}}(R)=$ $\beta(R)$.

Laser pulse transmission through rain and fog has been subject to intense research in recent years. As shown by many authors, both rain and fog consist of small water droplets. Scattering at a single droplet of water is modeled by scattering of an electromagnetic wave at a dielectric sphere exhibiting a diameter $D$ and a complex refraction index $\underline{n}_{W}$ which is depending on the wavelength. A typical value is $\underline{n}_{W}=1.323520+\mathrm{j} 5.150 \times 10^{-7}$ for $\lambda=905 \mathrm{~nm}$.

For a single, spherical water droplet, the extinction efficiency $Q_{\mathrm{EXT}}(D)$ and the backscattering efficiency $Q_{\mathrm{B}}(D)$ can be calculated using MIE's theory described in Bohren and Huffman (1983). Figure 3.5 shows the corresponding values for $Q_{\mathrm{EXT}}$ and $Q_{\mathrm{B}}$ as a function of droplet diameter D.

To model rain or fog, a statistical distribution of water droplets featuring different diameters $D$ can be used. The probability to hit a droplet of diameter $D$ is said to be $N(D)$. Assuming only single, non-elastic scattering, i.e. the light scattered by a water droplet is not scattered by another droplet and no energy is converted to other wavelength, the extinction coefficient $\alpha$ and the backscattering coefficient $\beta$ can be calculated to be:

$\alpha=\frac{\pi}{8} \int_{D=0}^{\infty} D^{2} Q_{\mathrm{EXT}}(D) N(D) d D$ 


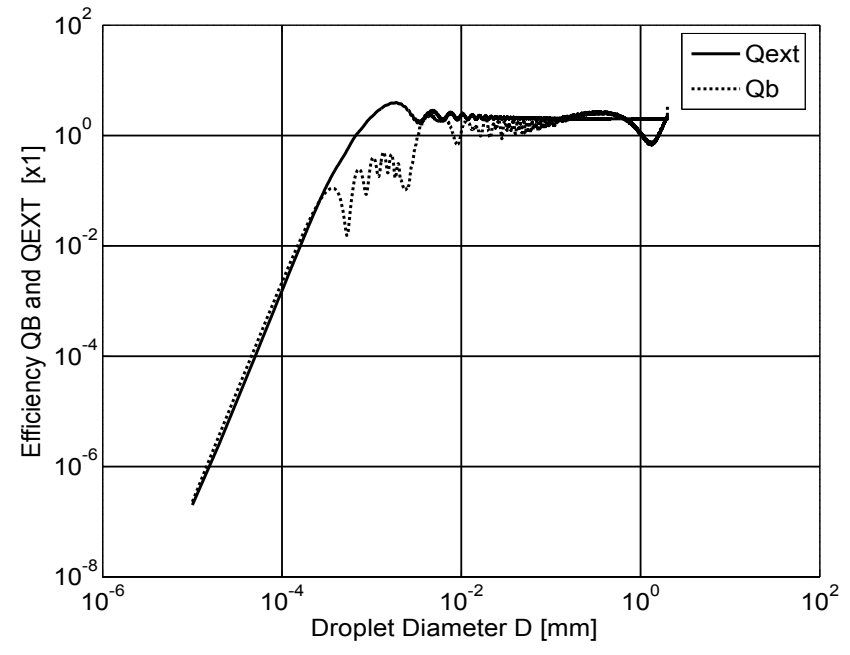

Fig. 3. Extinction efficiency $Q_{\mathrm{EXT}}(D)$ and backscattering efficiency $Q_{\mathrm{B}}(D)$ for a water droplet of diameter $D$ at $\lambda=905 \mathrm{~nm}$.

$\beta=\frac{\pi}{8} \int_{D=0}^{\infty} D^{2} Q_{\mathrm{B}}(D) N(D) d D$

In the past, many authors studied the distribution of droplet diameters for rain and fog resulting in different models for $N(D)$. Marshall and Palmer (1948) modeled the diameter of rain droplets as an exponential function of rain rate $R_{\mathrm{R}}$ which is expressed in $\mathrm{mm} \mathrm{h}^{-1}$ :

$N(D)=8000 \frac{1}{\mathrm{mmm}} \exp \left(-4.1 R_{\mathrm{R}}^{-0.21} D\right)$

As turned out during our investigations (see Adelsperger, 2008), this simple model does not hold true for laser radar pulse transmission due to the short measurement time in which large deviation from the exponential distribution (21) are likely. Feingold and Levin (1985) used a Log-normal distribution to improve the model of Marshall and Palmer (1948), however we found that using a Gamma distribution according to Deirmendjian (1969) provides most flexibility to model both rain and fog (see Isaac et al., 2001). In their model, four parameters $\alpha, \gamma, \rho$ and $r_{\mathrm{C}}$ describe the distribution $N(D)$ :

$N(D)=\frac{\gamma \rho b^{\frac{\alpha+1}{\gamma}}}{\Gamma\left(\frac{\alpha+1}{\gamma}\right)}\left(\frac{D}{2}\right)^{\alpha} e^{-b\left(\frac{D}{2}\right)^{\gamma}}$

with

$b=\frac{\alpha}{\gamma\left(D_{\mathrm{C}} / 2\right)^{\gamma}}$

In Eq. (22), $\Gamma(\cdot)$ denotes the Gamma function and $D_{C}$ denotes the droplet radius having maximum probability.
Table 1. Parameters of droplet size distribution in rain using a gamma-function model.

\begin{tabular}{lrrrr}
\hline Weather condition & $\rho\left[\mathrm{m}^{-3}\right]$ & $\alpha$ & $\gamma$ & $r_{\mathrm{C}}[\mathrm{mm}]$ \\
\hline Rain (coast) & 1000 & 1 & 0.5 & 0.05 \\
Rain (continental) & 1000 & 2 & 0.5 & 0.07 \\
\hline
\end{tabular}

Table 2. Parameters of droplet size distribution in fog using a gamma-function model.

\begin{tabular}{lrrrr}
\hline Weather condition & $\rho\left[\mathrm{cm}^{-3}\right]$ & $\alpha$ & $\gamma$ & $r_{\mathrm{C}}[\mu \mathrm{m}]$ \\
\hline Haze (coast) & 100 & 1 & 0.5 & 0.05 \\
Haze (continental) & 100 & 2 & 0.5 & 0.07 \\
Strong advection fog & 20 & 3 & 1.0 & 10.00 \\
Moderate advection fog & 20 & 3 & 1.0 & 8.00 \\
Strong spray & 100 & 6 & 1.0 & 4.00 \\
Moderate spray & 100 & 6 & 1.0 & 2.00 \\
Fog of type "Chu/Hogg" & 20 & 2 & 0.5 & 1.00 \\
\hline
\end{tabular}

Tables 1 and 2 show the parameters of the droplet size distribution for various types of rain and fog. In the automotive environment, however, the given distributions are strongly influenced by spray from other cars or trucks and thus tend to vary strongly from the given model. Although the given models are not accurate in these cases, they provide a good starting point for further investigations and a good first guess on the backscattered power levels and the transmission power loss factors to be expected.

\subsection{Snow}

Snowflakes may consist of single ice crystals or aggregates of ice crystals featuring varying mass densities ranging from $0.005 \mathrm{~g} \mathrm{~cm}^{-3}$ to $0.5 \mathrm{~g} \mathrm{~cm}^{-3}$ depending on temperature and various other conditions see Oguchi (1983); Rasmussen et al. (1999). The size distribution $N(D)$ of the snowflake aggregates can be modeled to be

$N(D)=N_{0} \exp (-\Lambda D)$.

depending on the precipitation rate $R_{\mathrm{P}}$ measured in millimeters of equivalent liquid water per hour, see Nebuloni and Capsoni (2008). Table 3 shows parameters of this distribution as determined by different authors.

Electromagnetic modeling of single snowflakes turns out to be very difficult due to their complex geometric shape and their strongly varying water content. Investigations were carried out by different authors modeling ice crystals as aggregates of dielectric spheres as investiged by Neshyba (2003), hexagonal slabs considered by Yang and Liou (1996) or using an extension to MIE's theory as done by Zhou et al. (2003). A rough estimation of the extinction coefficient $\alpha_{\text {Snow }}$ in case of dry and wet snow is given in Eqs. (25) and (26), respectively. 
Table 3. Parameters of size distribution of snowflakes.

\begin{tabular}{llcc}
\hline Author & Reference & $N_{0}$ & $\Lambda$ \\
\hline Gunn et al. & Gunn and Marshall (1957) & $7.6 \times 10^{3} \times R^{-0.87}$ & $2.55 \times R^{-0.48}$ \\
Sekhon et al. & Sekhon and Srivastava (1970) & $5.0 \times 10^{3} \times R^{-0.94}$ & $2.29 \times R^{-0.45}$ \\
\hline
\end{tabular}

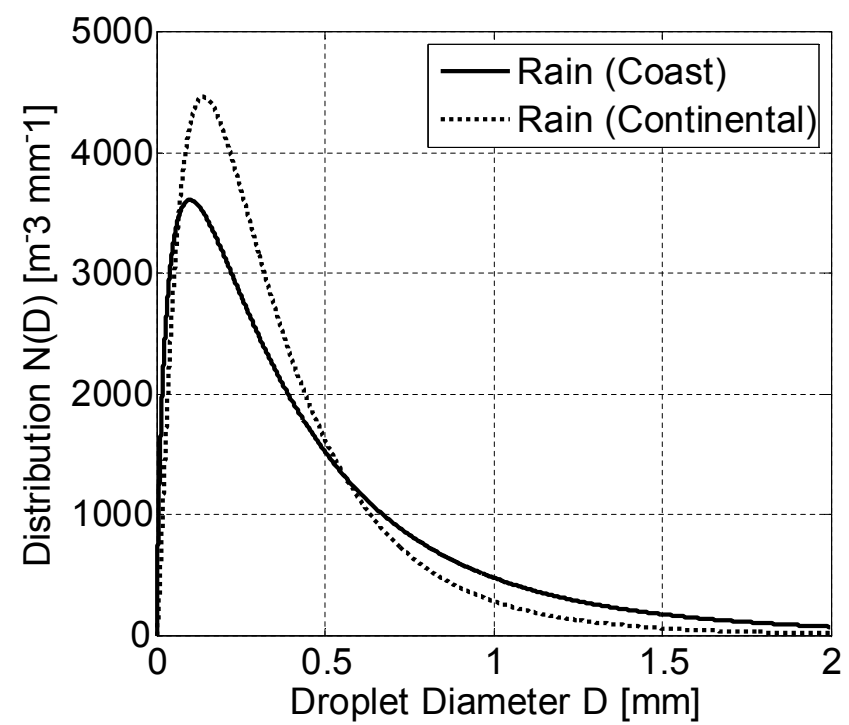

Fig. 4. Distribution of rain droplets according to model Eq. (22) with parameters of Table 1.

$\alpha_{\text {Snow,Dry }} \approx 15 \mathrm{~h} \mathrm{~mm}^{-1} \mathrm{~dB} \mathrm{~km}^{-1} R_{\mathrm{P}}+1 \mathrm{~dB} \mathrm{~km}^{-1}$

$\alpha_{\text {Snow, Wet }} \approx 2 \mathrm{~h} \mathrm{~mm}^{-1} \mathrm{~dB} \mathrm{~km}^{-1} R_{\mathrm{P}}-0.1 \mathrm{~dB} \mathrm{~km}^{-1}$

\subsection{Unwanted raw detection due to weather phenomena}

Backscattering from snowflakes or water droplets turns out to be a source of unwanted detections in automotive laser radar systems as explained in Sect. 2.6. In a critical distance range $0<R<R_{2}\left(R_{2}<10 \mathrm{~m}\right.$ in most sensor systems) where the crossover function $\xi(R)$ (see Eq. 10) is rising from 0 to 1 , the transmit beam power density is very high so snowflakes or water droplets acting as small but efficient reflectors are likely to generate false alarms. Although - at first glance these detections might be easily removed by means of tracking filters, the necessity for filtering introduces time delays or rises detection threshold levels while still not guaranteeing total removal of unwanted detections which are not tolerable in safety-critical vehicle functions like e.g. autonomous emergency braking. The situation becomes worse in case of coaxial beam configurations where $R_{2}=0$. The receive

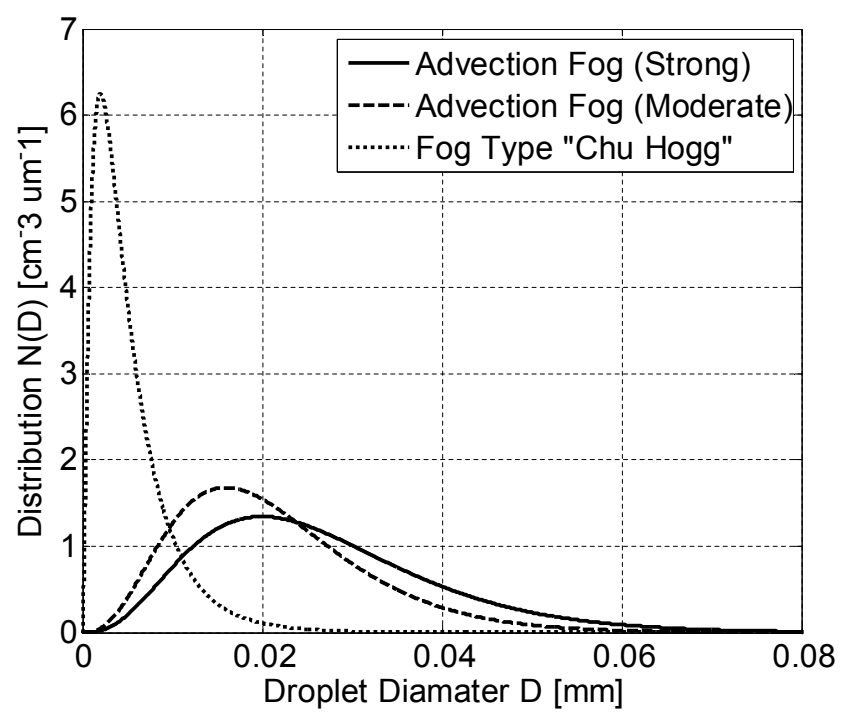

Fig. 5. Distribution of fog droplets according to model Eq. (22) with parameters of Table 2 .

power levels generated by snowflakes or water droplets in these cases are surprisingly high and tend to easily overload the optical receiver chain. Adaption of transmit power between pulses (inter pulse transmit power control) is a common cure, however leads to further reduction of the maximum detection range which is already low in case of heavy rain and snow anyway. Another approach to improve the situation is using a receiver architecture featuring enhanced dynamic range and using fast gain control mechanisms in every amplifier stage which, however, adds cost and complexity to the sensor.

During a winter-time measurement campaign, we determined the false alarm rate of a scanning laser radar featuring a coaxial beam configuration. As can be seen in Fig. 6, the large transmit power density of the laser radar and the high reflectivity of snowflakes generates a false alarm rate of up to $2.5 \mathrm{~s}^{-1} \mathrm{~m}^{-2}$ in a range of $5 \mathrm{~m}$ in front of the sensor. Using a bistatic beam configuration with properly designed opening angles $\gamma_{\mathrm{T} / \mathrm{R}}$ and TX/RX spacing $d$, the false-alarm rate can be reduced, however still will require careful attention. This is especially true if the sensor user does not have access to the raw detections of the system but has to rely on the analysis of tracked data during sensor evaluation. 


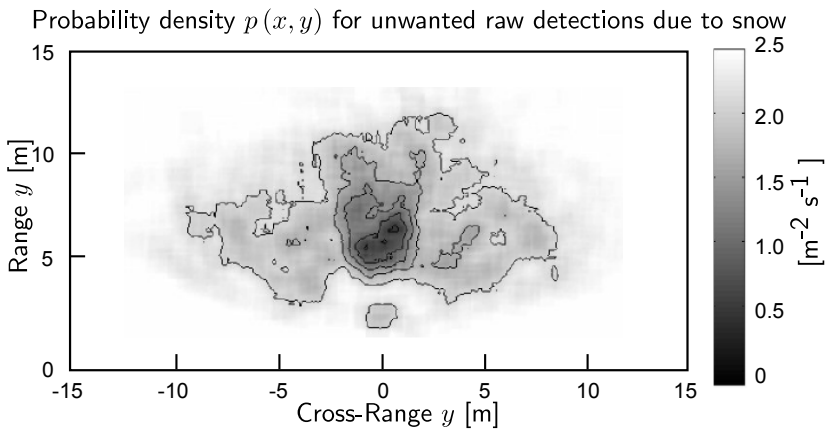

Fig. 6. Probability density $p(x, y)$ of unwanted raw target detections due to heavy snowfall, measured with an automotive laser radar system.

\subsection{Verification and conclusions on signal modeling}

The predicted laser radar return signals have been compared with real-world measurements in fog, rain and snow along with reference measurements with hard targets of known reflectivity. For hard laser radar reference targets (see Sect. 4.1), about 10-20\% absolute accuracy was reached. Long-term tests in fog showed very good agreement concerning the laser radar return signal (see Fig. 7). Residual deviations between measured data and predicted values are due to simplifications in modeling of the overlap function, the power homogeneity across the beam and the assumption of uniform fog distribution.

In practice, it turns out to be very hard to predict the local distribution functions of rain or fog which are strongly varying within the the field of view and over time. Moreover, experiments showed that the droplet distribution in the vicinity of the road surface is far from being uniform due to spray from other cars, large trucks or vans. In these cases, it might make sense to rely on pre-recorded data from previous test sessions and rather use the models to scale or extrapolate the signatures for different environmental conditions than to predict the laser radar returns from scratch.

\section{Conventional laser radar target simulation}

\subsection{Standard targets}

Road testing of a laser radar provides a realistic insight on the typical performance of the sensor, however comparison of the obtained test results between different sensor types or the same sensor with different software- and/or hardware configuration can only be done on a statistical basis and therefore needs a large amount of test data. To provide some trafficindependent figures of merit, the use of standard targets of known size and light reflectivity have turned out to be helpful. During our tests, we used aluminum plates featuring different types of coatings to realize standard targets of known
Table 4. Standard targets for laser radar evaluation.

\begin{tabular}{cccc}
\hline Target & Size & Reflectivity $\Gamma$ & Comment \\
\hline 1 & $0.8 \mathrm{~m}^{2}$ & $>4$ & High reflectivity target \\
2 & $0.8 \mathrm{~m}^{2}$ & 0.2 & Normal reflectivity target \\
3 & $0.8 \mathrm{~m}^{2}$ & 0.07 & Low reflectivity target \\
\hline
\end{tabular}

properties. Our reference targets all had a size of of $0.8 \mathrm{~m}^{2}$. To model low reflectivity targets, we used a coating featuring $\Gamma=0.07$, as normal reflection a coting featuring $\Gamma=0.2$ and as high reflecting target a coting providing $\Gamma=4$, see Table 4 .

\subsection{Measurement linearity}

Laser radars tend to introduce distance measurement errors depending on target reflectivity. This is due to optical overloading of the receiver chain or due to the detection process itself. This effect must be watched carefully during sensor evaluation. A simple test to check for the measurement linearity of a laser radar is using a plain, large concrete wall painted in white as a target. Under ideal conditions, the wall will be measured as a straight line of reflection points, one in each beam.

\subsection{Road testing under adverse weather conditions}

A classic approach to test the performance of laser radar systems under adverse weather conditions is doing road testing in heavy rain, dense fog or during snowfall. Although this approach enables accurate and realistic judgement of the sensor system's adverse weather performance, the results tend to depend heavily on the exact conditions present during test time and cannot be easily reproduced. Moreover, some weather conditions like heavy snowfall or dense fog can only be tested regionally or seasonally and thus can't be tested as required. Because of this, the improvement of the adverse weather performance of today's laser radar sensors is limited to the availability of reproducible test conditions. Overcoming this problem was the main goal of the optical laser radar target simulator which will be presented in Sect. 5 .

\subsection{Artificial simulation of rain}

Experiments were carried out to simulate rain under lab conditions using an artificial indoor rain simulator. The systems consists of 32 nozzles placed in a height of about $6 \mathrm{~m}$ distributing water over an area of $19 \times 12 \mathrm{~m}$. A highperformance pump system is enabling a maximum flow of water up to $6 \mathrm{~m}^{3} \mathrm{~h}^{-1}$ which equals a maximum rain rate of $27 \mathrm{~mm} \mathrm{~h}^{-1}$. The system proved to be quite helpful during sensor evaluation and comparison, however the spatial distribution of the artificially produced rain turned out to be unbalanced featuring pronounced peaks of rain density under the 


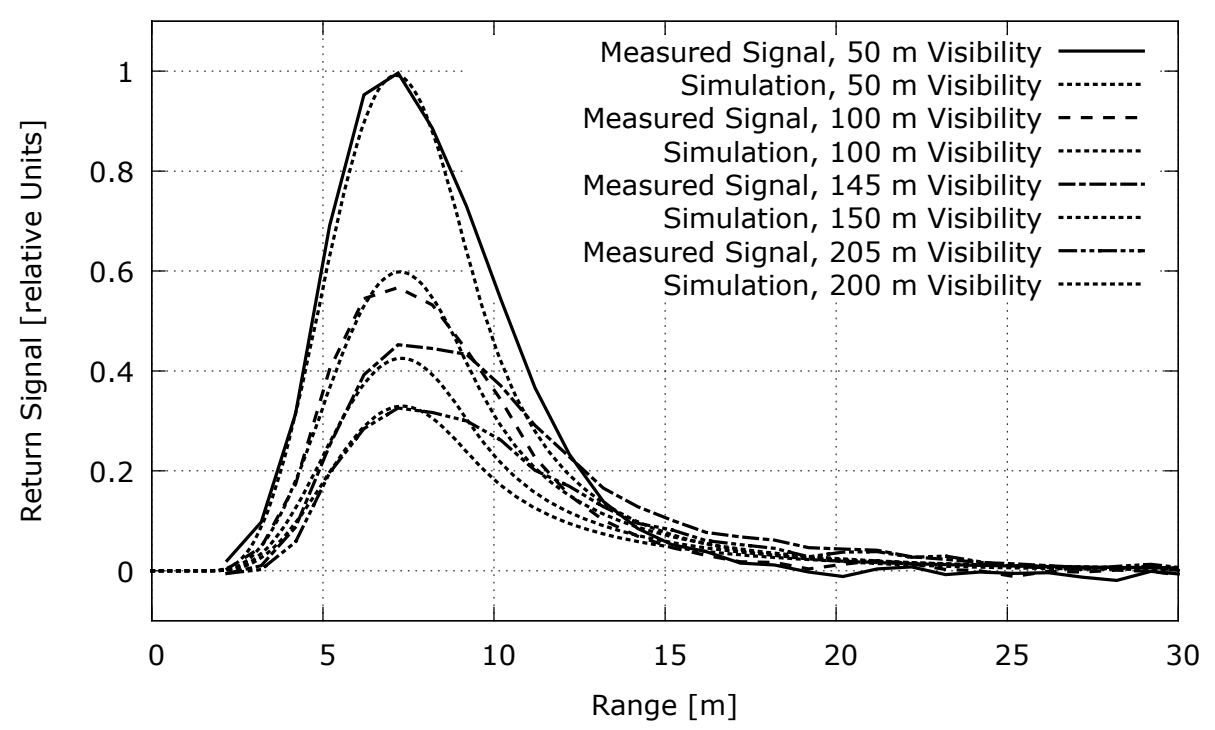

Fig. 7. Measured laser radar return signal in case of different visibility of $50 \mathrm{~m}, 100 \mathrm{~m}, 150 \mathrm{~m}$ and $200 \mathrm{~m}$ compared to numerically simulated

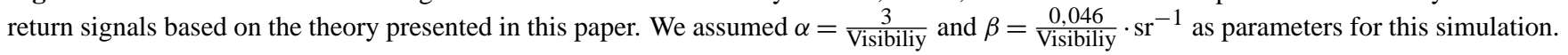

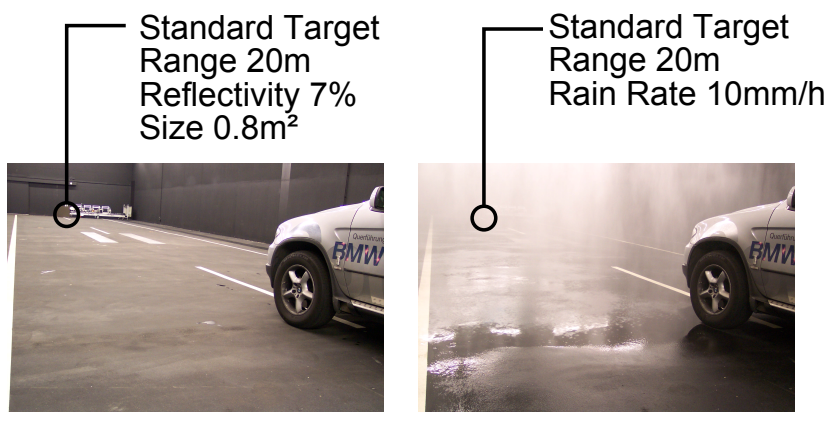

Fig. 8. Test session using indoor rain simulator without rain (left) and with a rain rate of $10 \mathrm{~mm} \mathrm{~h}^{-1}$ (right).

nozzles. Figure 8 shows a test session using the described indoor rain simulator, Fig. 9 shows results obtained using the rain simulator.

\section{Electro-optical laser radar target simulation}

\subsection{Introduction}

Frequently, sensor developers and users are faced with the task to evaluate software and hardware improvements of laser radar sensors. In this case, tests under adverse weather conditions usually cannot be done due to a lack of corresponding weather conditions (e.g. lack of snow or fog) during the evaluation phase. Moreover, if sensors from different manufacturers have to be evaluated, it turns out to be very hard to exactly reproduce the same environmental conditions for both sensors.

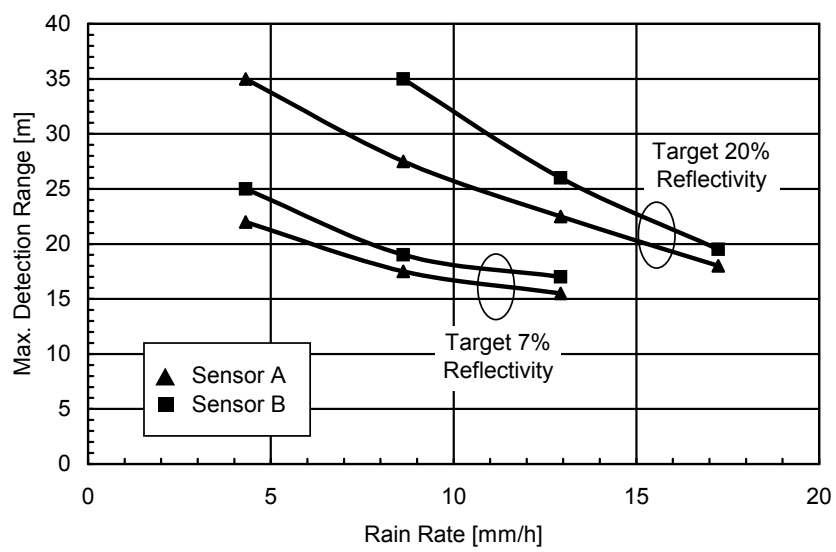

Fig. 9. Results of sensor tests using indoor rain simulator. The graphs show the maximum target detection range $R_{\mathrm{MAX}}$ as a function of rain rate for standard targets of $7 \%$ and $20 \%$ reflectivity and for sensors from two different manufacturers $\mathrm{A}$ and $\mathrm{B}$.

To overcome these difficulties, a novel electro-optical laser radar target simulator system (short: OSS) has been developed and used as an automotive laser radar target simulator. The OSS is capable to exactly reproduce the optical return signals measured by reference laser radars under adverse weather conditions by highly accurate replication of pulse shape, wavelength and power levels. It can handle multiple reflections in one sensor beam and might be extended to be used with scanning laser radar systems, too. With the OSS system, it became possible to measure weather performance enhancements due to hardware and/or software changes during the laser radar design process thus shortening the design cycle and improving the sensor quality. The test signals can 


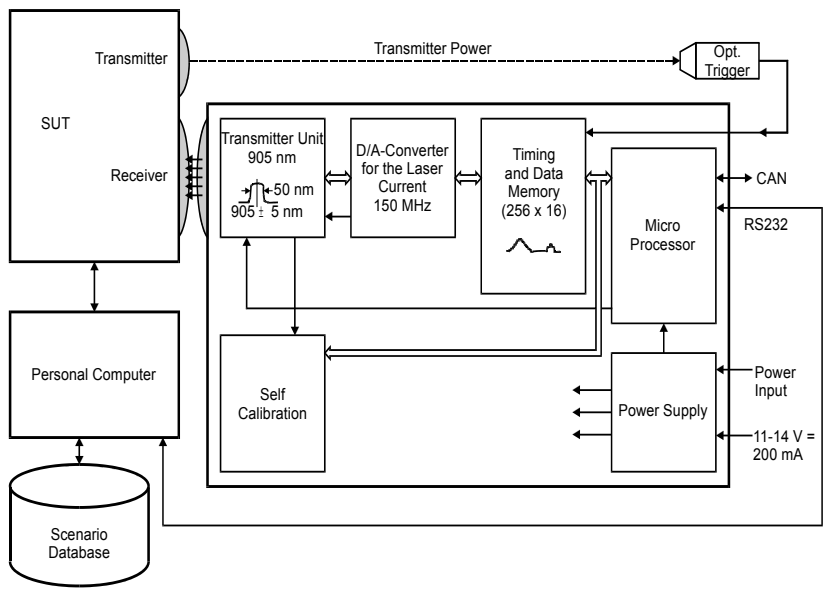

Fig. 10. Block diagram of an optical laser radar target simulator.

either be pre-recorded by the DUT itself or a by reference laser radar. Moreover, the test signals might be generated synthetically using the theory presented in Sect. 3. The system is not directly comparable to known millimeter-wave radar target generators (see e.g. Anritsu, 2009) since those only take signal delay and signal attenuation into account and cannot change the pulse's time-domain signature.

\subsection{Technical description}

Figure 10 shows a block diagram of the OSS. The DUT's transmit pulse is received by the optical trigger unit of the OSS. Upon reception, it triggers data transfer of a stored backscatter signal from the data memory of the OSS towards a DAC at a clock rate of $150 \mathrm{MHz}$. The output of the DAC drives a laser diode by means of a fast precision power amplifier. The backscatter data is either stored in a test computer or in the microprocessor unit of the OSS and can be repeated statically or dynamically with a maximum repetition rate of about $560 \mathrm{kHz}$ which is equal to a maximum target range of $250 \mathrm{~m}$ for the OSS simulation, taking an offset of $6 \mathrm{~m}$ due to trigger delay into account. The output power of the OSS transmitter unit is radiated through its output lens towards the DUT, constant illumination can be added to emulate disturbing background light. With an evaluation software, the backscatter signal output of the DUT can be recorded on the test computer and compared with the expected response of the DUT.

The OSS is capable of generating a calibrated power density of $0.5 \mathrm{nW} \mathrm{mm} m^{-2}$ to $2 \mu \mathrm{W} \mathrm{mm}^{-2}$ at the optical receiver of the DUT, featuring an dynamic range of $36 \mathrm{~dB}$.

The power density $S_{\mathrm{D}}$ on the DUT's photo detector generated by the OSS transmitter power density $S_{\mathrm{O}}$ is depending on the focal length $f_{\mathrm{D}}$ of the DUT's receiver, the diameter $D_{\mathrm{D}}$ of the DUT's lens and the focal length $f_{\mathrm{O}}$ of the OSS transmitter and its lens diameter $D_{\mathrm{O}}$, respectively. Moreover, as the output power of the OSS can be set to discrete values
Table 5. Key technical data of OSS.

\begin{tabular}{|c|c|}
\hline Criteria & Data \\
\hline Wavelength & $905 \pm 5 \mathrm{~nm}$ \\
\hline Bandwidth & $40 \mathrm{~nm}$ \\
\hline $\begin{array}{l}\text { Deviation of optical axis to } \\
\text { unit case }\end{array}$ & $< \pm 5 \mathrm{deg}$ \\
\hline $\begin{array}{l}\text { Focal length } f_{\text {OSS }} \text { of output } \\
\text { lens }\end{array}$ & $80 \mathrm{~mm}$ \\
\hline $\begin{array}{l}\text { Diameters of output lens } \\
D_{\text {OSS }}\end{array}$ & $38 \mathrm{~mm}$ \\
\hline Diffuser's aperture & $4 \mathrm{~mm}$ diameter \\
\hline $\begin{array}{l}\text { Spatial deviation of power } \\
\text { density }\end{array}$ & $\begin{array}{l} \pm 5 \% \text { in an area of } 2 \mathrm{~mm} \text { dia- } \\
\text { meter around the center of dif- } \\
\text { fuser's aperture }\end{array}$ \\
\hline $\begin{array}{l}\text { Minimum output power } \\
\text { density }\end{array}$ & $0.5 \mathrm{nW} \mathrm{mm}^{-2}$ \\
\hline $\begin{array}{l}\text { Maximum output power } \\
\text { density }\end{array}$ & $2 \mu \mathrm{W} \mathrm{mm}{ }^{-2}$ \\
\hline $\begin{array}{l}\text { Output power density quan- } \\
\text { tization }\end{array}$ & $5.2 \times 10^{-10} \mathrm{~W} \mathrm{~mm}^{-2} \mathrm{Bit}^{-1}$ \\
\hline Output power steps & 4096 (12 Bit quantization) \\
\hline Time discretization & $6.67 \mathrm{~ns}$ \\
\hline Trigger accuracy & $3.33 \mathrm{~ns}$ \\
\hline Optical trigger delay & $\approx 50 \mathrm{~ns}$ \\
\hline Range discretization & $1 \mathrm{~m}$ \\
\hline Accuracy of calibration & $\pm 10 \%\left(18\right.$ to $\left.24^{\circ} \mathrm{C}\right)$ \\
\hline Data interface & $\mathrm{RS} 232$ \\
\hline Power supply & 11-14 V/typ. $200 \mathrm{~mA}$ \\
\hline
\end{tabular}

in steps of $S_{q}$, it is controlled by a digital value $N$ taken from the signature memory ranging from 0 to 4095 . Equation (27) describes the corresponding relations:

$S_{D}= \begin{cases}N S_{q}\left(\frac{f_{O}}{f_{\mathrm{D}}}\right)^{2}\left(\frac{D_{\mathrm{D}}}{D_{\mathrm{O}}}\right)^{2} & \text { for } D_{\mathrm{D}}<D_{\mathrm{O}} \\ N S_{q}\left(\frac{f_{\mathrm{O}}}{f_{\mathrm{D}}}\right)^{2} & \text { for } D_{\mathrm{D}} \geq D_{\mathrm{O}}\end{cases}$

Figure 11 shows a picture of the first OSS prototype testing an automotive laser radar. To provide accurate calibration, a thermal secondary standard and a calibrated photo PIN diode receiver was used at an output power density of $2 \times 10^{-6} \mathrm{~W} \mathrm{~m}^{-1}$. The accuracy of both secondary standards was $\pm 2 \%$.

\subsection{Measured results}

To prove the basic functionality of the OSS, a 16-channel automotive laser radar was stimulated with both synthetic and pre-recorded real-world sensor signals.

With a reference laser radar sensor, the backscatter signal of a hard laser radar target located in fog was recorded (Fig. 12). This signal was replicated by the OSS and detected by a another laser radar in the lab. As can be seen (Fig. 13), 


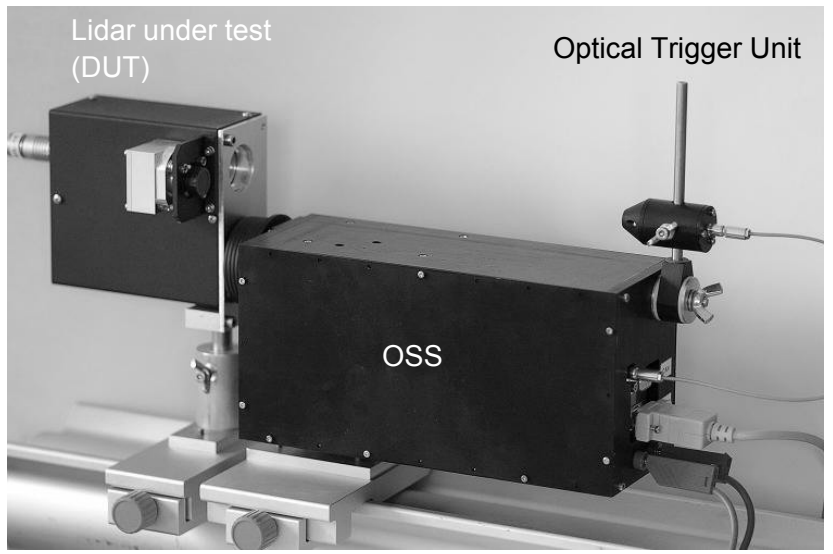

Fig. 11. OSS prototype hardware testing an automotive 16-beam laser radar.

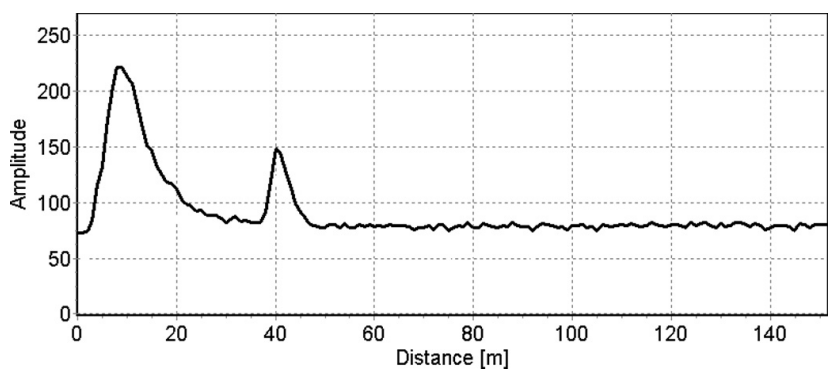

Fig. 12. Recorded signal of a target in fog used for sensor test.

very accurate simulation of the target located in foggy environment could be reached.

The examples show a limitation of the OSS target simulation: Since the OSS is triggered by the DUT's transmit laser pulse, a time delay is introduced for the simulated target signal. The delay is about $53 \mathrm{~ns}$ resulting in a virtual target shift of about $8 \mathrm{~m}$. Although this delay will be of minor importance for long-range laser radar target simulation, it limits possibilities for short range target simulation which are of special interest when considering influences of rain and fog. To overcome this limitation, different approaches might be taken:

- Changing the distance offset adjustment in the sensor.

- Electrical triggering of the OSS using a special electrical trigger output from the DUT or using a trigger signal derived from internal DUT trigger signals which typically are 45 to $55 \mathrm{~ns}$ ( 6 to $8 \mathrm{~m}$ ) prior to the actual transmit pulse output of the DUT's laser.

- Improvement of OSS' trigger circuitry minimizing delays within the OSS.

- Use of larger OSS trigger clock frequency.

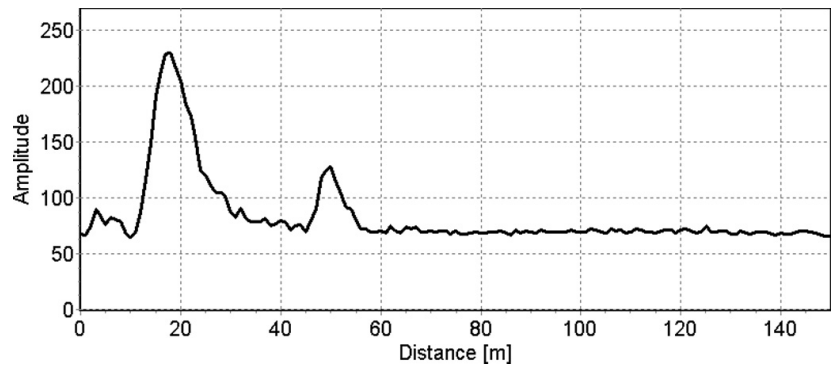

Fig. 13. Laser radar's response to stimulation according to Fig. 12.

The measured results showed that the OSS concept is a powerful approach for real-time HIL testing of automotive laser radar sensors. With the OSS concept, it is possible to test a laser radar's response to either real-world data obtained from test drives, or simulated data from hard or soft target models. Furthermore, a combination of measured data with numeric target modeling might be another option in many situations. Data once recored with a reference laser radar might be converted to signals stimulating other models of laser radar using Eq. (27).

\subsection{Next steps}

With a less-featured version of the OSS, an end-of-line testing and calibration of laser radars at the OEM's production line along with testing, adjustment and calibration is greatly simplified. To be compatible with laser radar sensors from different manufacturers featuring different wavelength, the transmit front-ends of the OSS might be easily changed. Complete sequences of recorded or simulated objects, including reduced visibility and rain or snow might be used to evaluate or compare laser radar based driver assistant systems' behavior using the OSS.

\section{Conclusions}

From laser radar theory and a model-based description of hard and soft laser radar targets, basic factors influencing the weather performance of modern automotive laser radar systems can be deduced. Based on this knowledge, improvement of laser radars' hardware and software is possible. To test the sensor performance improvements, three basic approaches are known: real world test drives under adverse weather conditions, tests with artificially induced weather phenomena (e.g. artificial rain) and electro-optical laser radar target simulation. Due to the drawbacks of first two approaches, a novel electro-optical laser radar target simulator system (short "OSS") has been developed, built and tested. The OSS prototype showed high accuracy in simulating laser radar performance in fog and proved to be very helpful during sensor optimization in the lab. Future improvements of the OSS concept should include a high-speed 
data interface enabling real-time, multi-channel playback of pre-recorded road scenarios. Generation of useful and accurate test data for rain, fog and snow should be based on prerecorded signatures scaled to the desired values using signal models or laser radar returns from snow, fog or rain. As experiments showed, signal modeling without calibration and scaling based on real world measurements might exhibit limited accuracy in the case of soft targets (e.g. fog) and should rather be used to model hard targets. Although of limited accuracy, soft target models turn out to be very useful for estimation of laser radar return signals during sensor system design.

\section{References}

Adelsperger, A.: Entwicklung innovativer Testverfahren für automobile LIDAR Sensoren, Diploma thesis, University of Applied Science, Munich, Germany, 2008.

Anritsu: ME7220A: Radar Test System (RTS) 76 to $77 \mathrm{GHz}$, http: //www.eltm.ru/store/Anritsu/ME7220A.pdf, 2009.

Bohren, C. F. and Huffman, D. R.: Absorption and Scattering of Light by Small Particles, John Wiley and Sons, 1983.

Darms, M. S., Rybski, P. E., Baker, C., and Urmson, C.: Obstacle Detection and Tracking for the Urban Challenge, IEEE Trans. Intell. Transp. Syst., 10, 475-484, 2009.

Deirmendjian, D.: Electromagentic Scattering on Spherical Polydispersions, Elsevier, 1969.

Fayad, F. and Cherfaoui, V.: Tracking objects using a laser scanner in driving situation based on modeling target shape, in: Proc. of the 2003 IEEE Intelligent Vehicles Symposium, Istanbul, Turkey, 2007.

Feingold, G. and Levin, Z.: The Lognormal Fit to Raindrop Spectra from Frontal Convective Clouds in Israel, J. Clim. Appl. Meteorol., 25, 1346-1363, 1985.

Grönwall, C., Steinvall, O., Gustafsson, F., and Chevalier, T.: Influence of laser radar sensor parameters on range-measurement and shape-fitting uncertainties, Opt. Eng., 46, 106201-1 - 106201$11,2007$.

Gunn, K. L. S. and Marshall, J. S.: The Distribution with Size of Aggregate Snowflakes, J. Meteorol., 15, 453-455, 1957.

Halldorsson, T. and Langerhoic, J.: Geometrical form factors for the lidar function, Appl. Optics, 17, 240-244, 1978.

IEC 60825-1:2007: Safety of laser products - Part 1: Equipment classification and requirements, in: IEC Standards, 2007.

Issa, B.: Optimierte Signalverarbeitung im Bereich der Fahrerassistenzsysteme, Diploma thesis, Technische Universität München, Munich, Germany, 2007.

Isaac, K. I., McArthur, B., and Korevaar, E.: Comparison of laser beam propagation at $785 \mathrm{~nm}$ and $1550 \mathrm{~nm}$ in fog and haze for optical wireless communications, Proc. SPIE Vol. 4214, Optical Wireless Communications III, pp. 26-37, 2001.

Kirchner, A. and Ameling, C.: Integrated Obstacle and Road Tracking using a Laser Scanner, in: Proc. of the 2000 IEEE Intelligent Vehicles Symposium, Dearborn (MI), USA, 2000.
Marshall, J. S. and Palmer, W.: The Distribution of Raindrops with Size, J. Appl. Meteorol., 5, 165-166, 1948.

Nebuloni, R. and Capsoni, C.: Laser Attenuation by Falling Snow, in: Proc. of the 2008 Symp. on Communication Systems, Networks and Digital Signal Processing, pp. 265-269, Graz, Austria, 2008.

Neshyba, S. P.: Representation of a nonspherical ice particle by a collection of independent spheres for scattering and absorption of radiation: 2. Hexagonal columns and plates, J. Geophys. Res., 108, 6.1-6.18, 2003.

Oguchi, T.: Electromagnetic Wave Propagation and Scattering in Rain and Other Hydrometeors, Proc. of the IEEE, 71(9), 10291078, 1983.

Owens, J. C.: Optical Doppler Measurement of Microscale Wind Velocity, Proc. IEEE, 57(4), 530-536, 1969.

Rasmussen, R. M., Vivekanandan, J., Cole, J., Myers, B., and Masters, C.: The estimation of snowfall rate using visibility, J. Appl. Meteorol., 38, 1542-1563, 1999.

Sassen, K. and Dodd, G.: Lidar crossover function and misalignment effects, Appl. Optics, 21, 3162-3165, 1982.

Sekhon, R. S. and Srivastava, R. C.: Snow size spectra and radar reflectivity, J. Meteorol., 27(2), 299-307, 1970.

Skolnik, M.: Radar Handbook, McGraw-Hill Publishing Company, 1990.

Smith, D., Hooper, R., and Garrett, I.: Receivers of optical communications: A comparison of avalanche photodiodes with PINFET hybrids, Optical and Quantum Electronics, 10, 293-300, 1978.

Spies, H. and Spies, M.: Advanced sensor technology boosts fast passenger safety to new system design, in: 8th International Symposium and Exhibition on Car Occupant Safety Systems, Karlsruhe, Germany, 2006a.

Spies, M. and Spies, H.: Automobile Lidar Sensorik: Stand, Trends und zukünftige Herausforderungen, Adv. Radio Sci., 4, 99-104, doi:10.5194/ars-4-99-2006, 2006b.

Steinvall, O., Chevalier, T., and Larsson, H.: Data collection and simulation of high range resolution laser radar for surface mine detection, Proc. SPIE 6214, pp. 85-102, 2006.

Thrun, S., Burgard, W., and Fox, D.: Probabilistic Robotics, MIT Press, 2005.

Walchshäusl, L., Lindl, R., Vogel, K., and Tatschke, T.: Detection of road Users in Fused Sensor Data Streams for Collision Mitigation, in: Proc. Advanced Microsystems for Automotive Applications AMAA 2006, pp. 53-65, Berlin, Germany, 2006.

Yang, P. and Liou, K. N.: Geometric-optics - integral-equation method for light scattering by nonspherical ice crystals, Appl. Optics, 35, 6568-6584, 1996.

Zhao, Y., Lea, T. K., and Schotland, R. M.: Correction function for the lidar equation and some techniques for incoherent $\mathrm{CO}_{2}$ lidar data reduction, Appl. Optics, 27, 2731-2740, 1988.

Zhou, X., Shusun, L., and Stamnes, K.: Geometrical-optics code for computing the optical properties of large dielectric spheres, Appl. Optics, 42, 4295-4396, 2003. 